Revista Electrónica de Ciencias de la Educación, Humanidades, Artes y Bellas Artes

Año IV. Vol IV. N ${ }^{\circ}$ 7. Enero - Junio. 2021

Hecho el depósito de Ley: FA2018000022

ISSN: 2665-0282

FUNDACIÓN KOINONIA (F.K).

Santa Ana de Coro, Venezuela

Danny Rafael Rosero-Guanotásig; Ricardo Patricio Medina-Chicaiza

http://dx.doi.org/10.35381/e.k.v4i7.1175

\title{
Gamificación: Estrategia para la enseñanza de operaciones elementales de matemáticas
}

Gamification: Strategy for teaching elementary math facts

Danny Rafael Rosero-Guanotásig

drosero90@gmail.com

Pontificia Universidad Católica del Ecuador, Ambato

Ecuador

https://orcid.org/0000-0002-5706-7578

Ricardo Patricio Medina-Chicaiza

patricio medina@hotmail.com

Pontificia Universidad Católica del Ecuador, Ambato

Ecuador

https://orcid.org/0000-0002-2736-8214

Recepción: 26 de noviembre 2020

Revisado: 20 de diciembre 2020

Aprobación: 01 de febrero 2021

Publicación: 20 de febrero 2021 


\title{
RESUMEN
}

La gestión educativa constituye un desafío en estos tiempos de cambio, gracias al apoyo de las tecnologías y de herramientas tecnológicas se estimulan los ambientes de formación brindando a los niños y jóvenes mediante el juego nuevas formas de enseñanza-aprendizaje. El propósito general de la investigación es analizar la importancia de la gamificación como estrategia en la enseñanza de las matemáticas de una Unidad Educativa de Educación Básica. El estudio es una investigación descriptiva documental con diseño bibliográfico, ya que se utilizaron técnicas de exploración documental para fundamentar la estrategia educativa como la gamificación y la enseñanza de las matemáticas. Se utilizó la técnica ladov propuesta por (Kuzmina, 1970) para validar la estrategia didáctica de gamificación a través de una encuesta de satisfacción. Se concluye que para su aplicación es pertinente una capacitación a los docentes, donde se familiaricen con el entorno de la herramienta y sus diferentes funciones.

Descriptores: Juego de simulación; programa informático didáctico; proceso de aprendizaje; gestión de la educación; red informática. (Palabras tomadas del Tesauro UNESCO).

\begin{abstract}
Educational management constitutes a challenge in these times of change, thanks to the support of technologies and technological tools, training environments are stimulated by offering children and young people through play new forms of teaching-learning. The general purpose of the research is to analyze the importance of gamification as a strategy in the teaching of mathematics in an Educational Unit of Basic Education. The study is a descriptive documentary research with bibliographic design, since documentary exploration techniques were used to support the educational strategy such as gamification and the teaching of mathematics. The ladov technique proposed by (Kuzmina, 1970) was used to validate the didactic gamification strategy through a satisfaction survey. It is concluded that for its application a training for teachers is pertinent, where they become familiar with the environment of the tool and its different functions.
\end{abstract}

Descriptors: Simulation game; educational computer program; learning process; management of education; computer network. (Words taken from the UNESCO Thesaurus). 


\section{INTRODUCCIÓN}

El proceso de enseñanza-aprendizaje constituye una tarea noble y significativa cuyo principal fin es la formación integral de buenos ciudadanos en medio de un contexto social en constante movimiento, los Estados mediante su poder ministerial delega ante los ministerios respectivos la organización, planificación y ejecución de estrategias que garanticen la buena marcha de las actividades planteadas. En este sentido el Ministerio de Educación del Ecuador, plantea una visión crítica a la pedagogía, ya que promueve el aprendizaje significativo por medio de la interpretación y resolución de problemas, de esta manera los estudiantes deberán alcanzar la metacognición (Ministerio de Educación, 2020). Por tanto, en el País ecuatoriano dentro de los lineamientos de la Constitución y concretamente en el Plan Nacional para el Buen Vivir 2017 - 2021 (Constitución de la República del Ecuador, 2017), se plantea en el objetivo 7 lo siguiente: "incentivar una sociedad participativa, con un Estado cercano al servicio de la ciudadanía, y enmarcados en los lineamientos de la Ley Orgánica de Educación Intercultural (Reglamento General a la Ley Orgánica de Educación Intercultural, 2017) que abarca los subniveles: Preparatoria, Básica media, Básica superior; en la cual comprende la Básica Elemental que hace referencia a 2do, 3er y 4to de básica enfocada a estudiantes de 6 a 8 años, donde una de las asignaturas es Matemática, entre otras. Se busca impulsar la participación activa de los equipos educativos para lograr los objetivos académicos en las instituciones educativas y su entorno. Por ello el órgano encargado de la orientación y apoyo en materia educativa busca implementar acciones que contribuyan al desarrollo de la gestión educativa, al respecto el Ministerio de Educación del Ecuador (2012): establece:

El principal propósito de los estándares es orientar, apoyar y monitorear la acción de los actores del sistema educativo hacia su mejora continua. Adicionalmente, ofrecen insumos para la toma de decisiones de políticas públicas para la mejora de la calidad del sistema educativo. 
Estas mejoras deben facilitar la búsqueda de nuevas estrategias y herramientas que contribuyan a un mejor desarrollo de los niños, adolescentes y jóvenes en proceso de formación. Las exigencias actuales de la era tecnológica obligan a los docentes a innovar permanentemente, volviéndose imperecedera la incorporación de estrategias que respondan adecuadamente a los procesos formativos y que promuevan la colaboración, criticidad y conocimiento. (Holguín-García, Holguín-Rangel, \& García-Mera, 2020, p.72) En este sentido, de acuerdo al informe del censo 2017, presentado por el INEC, en el país existe un incremento del porcentaje de hogares con acceso a internet del $14,7 \%$ a nivel nacional, 15,2\% en el área urbana, y 11,3\% en el área rural; según esta importación esto constituye las condiciones optimistas en cuanto a entornos tecnológicas favorables para ser utilizado en diversos campos, uno de ellos la educación. (Instituto Nacional de Estadísticas y Censos, 2017). Esta ventaja competitiva contribuye a utilización de las tecnologías, para una innovación en el campo educativo, al respecto Macanchí-Pico, Bélgica-Marlene, \& Campoverde-Encalada, (2020) manifiestan que la innovación, en el campo educativo es apreciada como una estrategia para favorecer en logro de los objetivos institucionales y penetrar en todos los ámbitos, proceso y espacio educativo, estableciendo los escenarios para desarrollar una nueva cultura. Favoreciendo la producción de nuevas formas de enseñar que incentive en los estudiantes el interés por sus materias. En este mismo orden de ideas, Farías y Pérez (2010) afirman que motivar a los estudiantes de la presente época es una tarea difícil que actualmente enfrentan muchos docentes, sobre todo en el área de las llamadas ciencias exactas, como las Matemáticas; cuyo aprendizaje resulta complejo e incluso agotador para la mayoría de los estudiantes. Una alternativa para lograr la atención de los estudiantes es adoptar estrategias de juegos, que en el ámbito académico se las conoce como Gamificación (Prieto et al., 2014); que se convierte en una línea de acción lúdica; con el afán de mejorar la calidad de la enseñanza y aprendizaje de la Matemática. En este sentido, la Organización de las Naciones Unidas para la Educación, la Ciencia y la Cultura (Unesco, 
2014) promueven el uso del juego, no sólo para hacer más eficaz el proceso pedagógico, sino también para alcanzar el interés, el disfrute y la motivación que el estudiante necesita.(Gilbert \& Sarah, 1982). Sin embargo, de acuerdo a información recolectada mediante una encuesta a estudiantes de quinto año de Educación Básica, detallaron que no utilizan estrategias didácticas para el aprendizaje y comprensión de operaciones elementales en el área de matemáticas, de allí la importancia de indagar sobre las herramientas disponibles que faciliten el estudio y compresión de esta materia. Por todo lo planteado la investigación tuvo como objetivo general analizar la importancia de la gamificación como estrategia en la enseñanza de las matemáticas de una Unidad Educativa de Educación Básica.

\section{METODOLOGÍA}

El estudio es una investigación descriptiva documental con diseño bibliográfico, el enfoque fue cuantitativa, ya que se utilizaron técnicas de exploración documental para fundamentar la estrategia educativa como la gamificación en beneficio de la enseñanza de las matemáticas. Desde la perspectiva del análisis sintético permitió el estudio de la información recopilada, se empleó como instrumento de investigación la ficha o guía de análisis documental, lo que contribuyó a extraer las ideas y contrastarlas. Así como investigaciones, artículos científicos y datos estadísticos. Así mismo, posterior a la evaluación de varias plataformas tecnológicas, se utilizó la herramienta Classcraft (2020) para insertar contenido de la unidad quinta del libro de matemática para quinto año de educación básica, como en el artículo de García et al (2018); así como la herramienta Genial.ly (2020) propuesta por García y Neira (2017).

Cabe destacar que, se utilizó la técnica ladov propuesta por (Kuzmina, 1970) para validar la estrategia didáctica de gamificación a través de una encuesta de satisfacción que incluye tres preguntas cerradas de opción múltiple, las cuales dan paso al Índice de Satisfacción Individual (ISI) y al aplicar la fórmula se obtiene el Índice de Satisfacción 
Grupal (ISG). De esta representación, dio como resultado la respuesta de diez docentes del nivel de Educación Básica.

\section{RESULTADOS Y DISCUSIÓN}

\section{Visión del uso de la gamificación en el aprendizaje}

El uso y la utilización de la gamificación como estrategia de impacto en públicos determinados, fue el sector empresarial los pioneros en ponerlo en operación, donde fidelizan a sus clientes mediante artículos de colección. Por otra parte, las aerolíneas ganaban la lealtad de sus usuarios con sus programas de millas, a esta altura la educación no podía quedar excluida de esta ola innovativa; donde la experiencia lúdica toma gran importancia al trasladar las emociones y sensaciones de los videojuegos al espacio educativo.

Por otro lado, en cuanto a los límites de aprendizaje según Hammer y Lee (2011), mencionan que la gamificación desvanece los aprendizajes formales e informales, por tanto, se proyecta a los estudiantes hacia una diferente forma de conocimiento. La palabra gamificación se asimila como el uso del entono, las reglas y roles de personajes que manejan los juegos enfocados hacia la formación. Así, el valor agregado que aporta es en la habilidad para generar una nueva experiencia durante el aprendizaje; puesto que recoge elementos como (monedas, trofeos, insignias, medallas, poderes) y los destina hacia un entorno análogo del juego, en este caso concierne al salón de clase.

Existen varias experiencias desarrolladas mediante la gamificación, por ello existe un historial de la literatura sobre estas prácticas, donde en su gran mayoría hay resultados afirmativos (Hamari et al., 2014). La forma de educar a través de la ludificación (gamificación) es algo que se lleva a cabo de manera habitual, esta práctica se lleva a cabo por cuantiosos maestros a nivel mundial. Se ha comentado en varias ocasiones, en el cual la rutina del juego en las aulas constituye una destreza muy frecuente utilizadas 
por los docentes del nivel general básica; a su vez no es nada nuevo en la enseñanza aprendizaje tanto como para el bachillerato o en la universidad.

En tal sentido, la gamificación conlleva a la mejora en las prácticas de enseñanzaaprendizaje en las distintas etapas educativas, como lo planteó Aguilera et al. (2020) en su trabajo de investigación al evidenciar el gran potencial de la gamificación, pues generó gran interés en sus alumnos al impartir clases. En concordancia, Mallitasig y Freire (2020) tras aplicar la gamificación durante una unidad del plan de clases, afirman que sus estudiantes alcanzaron grandes aprendizajes significativos.

Así mismo, en otros niveles de formación es interesante como a nivel superior, la ludificación enganche los intereses y el estímulo de los estudiantes; en consecuencia, contribuya al incremento del nivel de responsabilidad en la formación como proceso. (Ardila, 2019). Entonces, para un ideal plan de gamificación, lo apropiado es potenciar herramientas que se ejecuten de manera fácil para optimizar la enseñanza, captar el interés, conseguir las ventajas de la red informática y en si toda la tecnología en general (Sánchez et al., 2020).

Por otro lado, Classcraft es una plataforma web enfocada a involucrar el uso de la gamificación en las aulas y contribuir a la optimización de la enseñanza. En resumen, aporta una serie de elementos, entornos y hasta varios comportamientos que de manera general aparecen en videojuegos para ejecutarlos en una o en muchas asignaturas. Como resultado, actúa como columna vertebral durante las lecciones, pruebas o exámenes, y a la misma vez brinda recompensas que se adquieren por el cumplimiento de las mismas, también se traslada a la vida real para así motivar aún más a los estudiantes (Mora y Camacho 2019).

Durante el desarrollo del presente trabajo se tomó en consideración los estándares de (Garcia et al., 2018), el cual manifiesta como primer paso que atrae el interés de los alumnos, como segundo paso explora actividades prácticas, el tercero explica los contenidos, el cuarto paso es donde se refuerza el conocimiento adquirido, el último paso 
conlleva una evaluación a los alumnos. No obstante, el autor manifiesta de manera general y no profundiza ningún proceso. Por otro lado, los autores Mora y Camacho (2019) propone tres fases, la fase inicial compuesta de la ambientación del aula, la segunda fase es el desarrollo, y su última fase la evaluación.

A continuación, se muestra el resultado obtenido de la revisión documental-bibliográfica sobre la gamificación, mediante una matriz en el mismo se observa el empleo de diversas herramientas lúdicas manejadas por los docentes que transforman el aprendizaje en actividades de emular o simular, por esta razón se las consideran como estrategias didácticas. En la tabla 1 se visualizan las actividades innovadoras más utilizadas de la gamificación en diversos escenarios educativos.

\section{Tabla 1.}

Matriz sobre la revisión documental sobre la gamificación desde la visión de varios autores.

\begin{tabular}{|c|c|c|c|}
\hline Autor & Año & Estrategia & Sistemática \\
\hline $\begin{array}{l}\text { Valda \& } \\
\text { Arteaga }\end{array}$ & 2015 & Plataforma CER & $\begin{array}{l}\text { El docente además de insertar contenido en la } \\
\text { plataforma, introduce insignias para motivar a } \\
\text { los estudiantes conformen alcancen los logros } \\
\text { esperados. }\end{array}$ \\
\hline $\begin{array}{l}\text { Torres, } \\
\text { et al. }\end{array}$ & 2018 & $\begin{array}{l}\text { Plataformas e- } \\
\text { learning } \\
\text { Duolingo } \\
\text { Babble aprende } \\
\text { idiomas } \\
\text { Edmodo } \\
\text { English } \\
\text { Aprende inglés con } \\
\text { Aba } \\
\text { Aprende inglés con } \\
\text { Wlingua } \\
\text { Classdojo }\end{array}$ & $\begin{array}{l}\checkmark \\
\text { Se validó los indicadores mediante un estudio } \\
\text { Delphi con expertos } \\
\checkmark \text { Se procede a realizar varios grupos de } \\
\text { estudiantes. } \\
\checkmark \text { EL docente selecciona una aplicación para } \\
\text { cada grupo de alumnos } \\
\text { Al finalizar el docente mide el grado de } \\
\text { interacción con la aplicación asignada, y el } \\
\text { desempeño alcanzado. } \\
\text { Este resultado permite al docente considerar } \\
\text { que aplicación puede ser considerada como } \\
\text { estrategia pedagógica. }\end{array}$ \\
\hline $\begin{array}{l}\text { Díez, } \\
\text { Bañeres \& } \\
\text { Serra }\end{array}$ & 2017 & Aplicación propia & 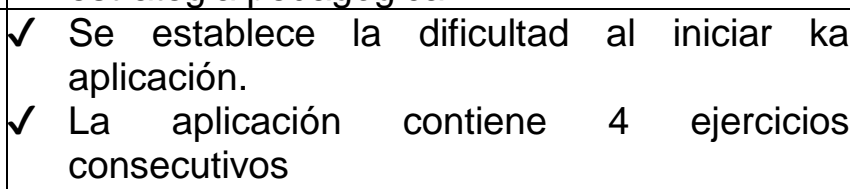 \\
\hline
\end{tabular}


Revista Electrónica de Ciencias de la Educación, Humanidades, Artes y Bellas Artes

Año IV. Vol IV. N $^{\circ}$. Enero - Junio. 2021

Hecho el depósito de Ley: FA2018000022

ISSN: 2665-0282

FUNDACIÓN KOINONIA (F.K).

Santa Ana de Coro, Venezuela

Danny Rafael Rosero-Guanotásig; Ricardo Patricio Medina-Chicaiza

\begin{tabular}{|c|c|c|c|}
\hline & & & $\begin{array}{l}V \text { Conforme realice de manera exitosa los retos } \\
\text { la aplicación le asigna puntos de experiencia, } \\
\text { caso contrario se le penaliza por cada } \\
\text { equivocación. } \\
\text { Al superar un nivel se almacena a manera de } \\
\text { ranking los puntajes de forma descendente de } \\
\text { todos los alumnos. }\end{array}$ \\
\hline Navarro & 2017 & Plataforma Kahoot & 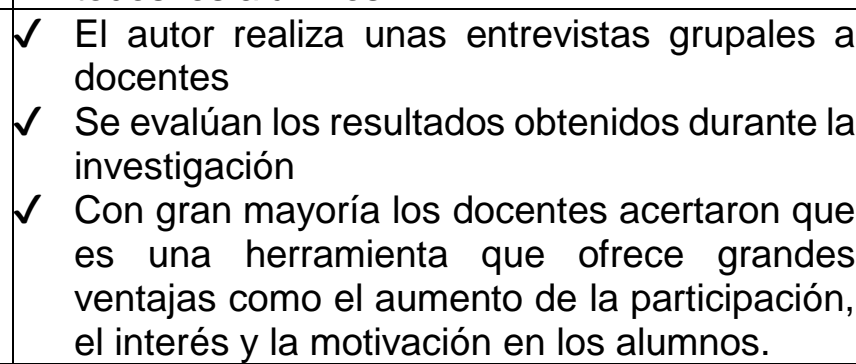 \\
\hline Corchuelo & 2018 & $\begin{array}{l}\text { Plataforma } \\
\text { Classdojo }\end{array}$ & 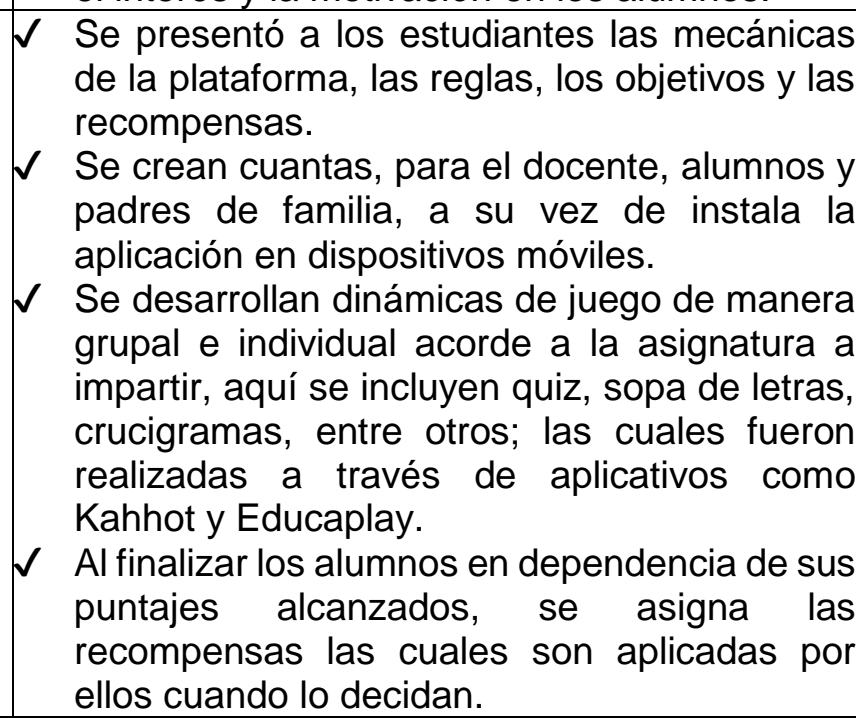 \\
\hline $\begin{array}{l}\text { García, } \\
\text { et al. }\end{array}$ & 2018 & $\begin{array}{l}\text { Classcraft } \\
\text { Alexia }\end{array}$ & $\begin{array}{l}\checkmark \text { El docente a través de un diario recopila la } \\
\text { actitud, el interés y los comentarios de los } \\
\text { alumnos. } \\
\checkmark \text { EL docente da un seguimiento a cada } \\
\text { estudiante en la plataforma institucional } \\
\text { Alexia. } \\
\checkmark \begin{array}{l}\text { Se realiza un registro de toda la } \\
\text { documentación como pruebas, tareas, } \\
\text { ejercicios. }\end{array}\end{array}$ \\
\hline $\begin{array}{l}\text { Mora y } \\
\text { Camacho }\end{array}$ & 2019 & Classcraft & $\begin{array}{l}\text { El docente a través del uso la plataforma } \\
\text { Classcraft pretende impulsar el trabajo en } \\
\text { equipo a través la cooperación de pequeños }\end{array}$ \\
\hline
\end{tabular}




\begin{tabular}{|c|c|c|c|}
\hline & & & $\begin{array}{l}\text { grupos, por otro, que los alumnos logren } \\
\text { apreciar sus cualidades individuales. }\end{array}$ \\
\hline $\begin{array}{l}\text { González, } \\
\text { et al. }\end{array}$ & 2020 & $\begin{array}{l}\text { Plataforma Khan } \\
\text { Academy }\end{array}$ & 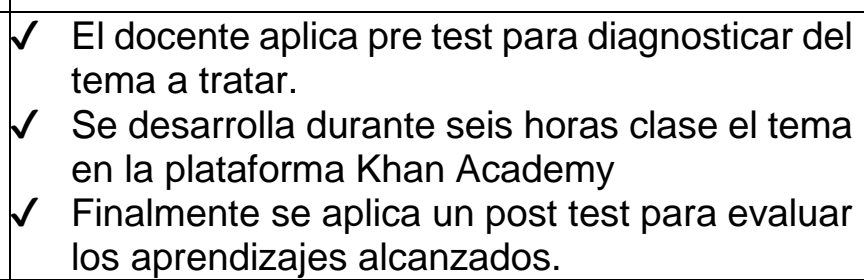 \\
\hline $\begin{array}{l}\text { Aguilera, } \\
\text { et al. }\end{array}$ & 2020 & $\begin{array}{l}\text { Plataforma } \\
\text { Classdojo } \\
\text { Aplicación móvil: } \\
\text { Series Lógicas de } \\
\text { Lucas }\end{array}$ & $\begin{array}{l}\checkmark \text { El docente explica sobre el contenido de la } \\
\text { clase. } \\
\checkmark \text { Cada estudiante realiza la actividad en la } \\
\text { aplicación Series Lógicas de Lucas. } \\
\checkmark \text { El docente registra los aciertos, el tiempo y los } \\
\text { puntos obtenidos para cada alumno y realiza } \\
\text { un seguimiento en la plataforma Classdojo. }\end{array}$ \\
\hline $\begin{array}{l}\text { Cruz \& } \\
\text { Cabero }\end{array}$ & 2020 & $\begin{array}{l}\text { Plataforma Quizizz } \\
\text { Plataforma Edmodo } \\
\text { (juego de triángulos) }\end{array}$ & 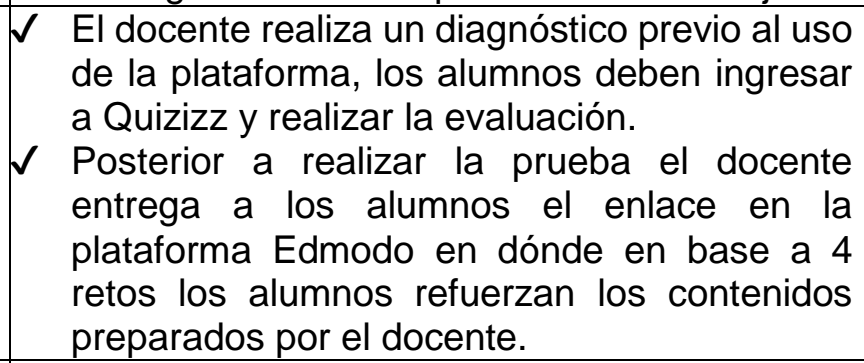 \\
\hline $\begin{array}{l}\text { Mallitasig } \\
\text { \& Freire }\end{array}$ & 2020 & $\begin{array}{l}\text { Plataforma Kahoot } \\
\text { Aplicación móvil } \\
\text { Plickers }\end{array}$ & $\begin{array}{l}\checkmark \text { El docente desarrollo con sus estudiantes en } \\
\text { la plataforma Kahoot una unidad completa. } \\
\checkmark \begin{array}{l}\text { Con la aplicación móvil Plickers evaluó los } \\
\text { resultados alcanzados. }\end{array}\end{array}$ \\
\hline
\end{tabular}

Fuente: Elaboración propia (2021).

Se observa en la matriz de la figura 1, la gran acogida y utilización de esta práctica se lleva a cabo maestros a nivel mundial, lo que demuestra el gran apoyo que brinda en desarrollo de estrategias pedagógicas en beneficios de los niños y jóvenes estudiantes. De acuerdo con Kob (1974), citado por Rodríguez-Cepeda (2017:54); consideró que la experiencia se refiere a toda serie de actividades que permiten aprender, considerando que el aprendizaje está condicionado a la experiencia vivida y consta de cuatro etapas que incluyen: la experiencia concreta, una observación reflexiva, la conceptualización abstracta y una experimentación activa. En este sentido, se propone trabajar mediante 
cuatro fases para la didáctica de gamificación: introducción, descubrimiento de contenidos, desarrollo de contenidos, evaluación de contenidos, las cuales se muestran a continuación:

\section{Primera fase. Introducción}

Este este primer apartado se explican las instrucciones, el uso de Classcraft, y se entrega los accesos hacia la plataforma. Hay dos documentos generados de forma automática cuando se crean los usuarios, estos archivos contienen las reglas, deberes y derechos que les otorga el uso de esta plataforma. Finalmente, hay otro documento donde se redacta un pacto con el estudiante, en donde se compromete en llegar hasta el final de los contenidos planificado por su docente.

\section{Figura 1.}

Introducción.

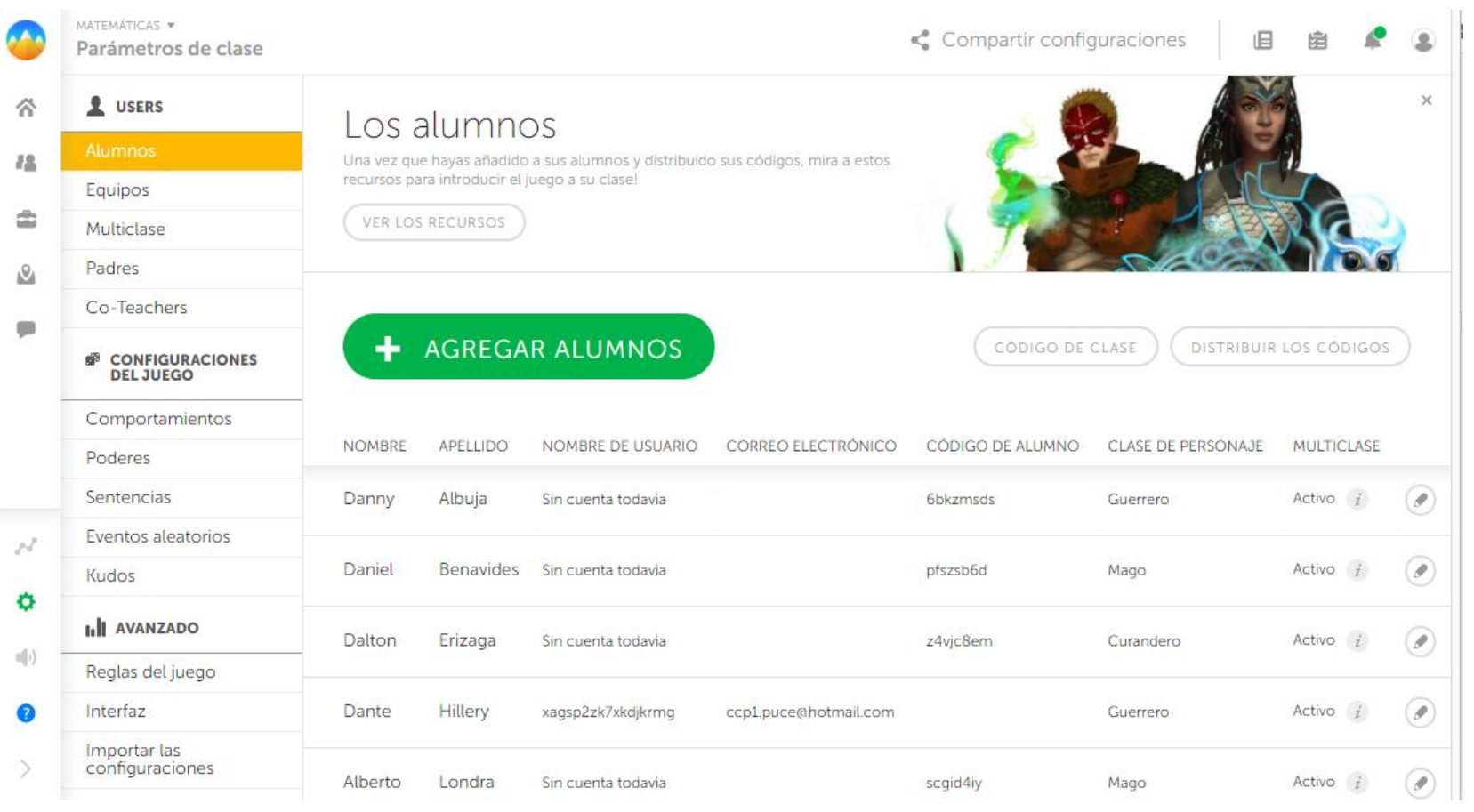

Fuente: https://game.classcraft.com/ 
A cada alumno se asigna un usuario y una contraseña, posterior a ello la plataforma solicita ingresar el rol del personaje creado, este puede ser: curandero, mago o hechicero; cada personaje posee poderes diferentes los cuáles a futuro le permite usar de forma individual o grupal cuando el docente imparta sus clases. Conforme las clases avancen, el alumno podrá equipar a su personaje con múltiples accesorios como cambios de ropa, mejoras de su armadura o incluso adquirir una mascota.

Todo aquello que complemente al uso de la plataforma influye de manera gradual en la asimilación de contenidos, despierta el interés en grande hacia los estudiantes para así realizar las actividades y tareas que a posterior se detalla en la siguiente fase. Cabe destacar que, tanto como los alumnos y los docentes se observan cambios, puesto que en sus estudiantes la predisposición hacia aprendizaje se incrementa. Lo mismo ocurre en el maestro, quien se verá más entusiasmado, es decir, permite una generación de contenido más amplia y didáctica.

\section{Segunda fase. Descubrimiento de contenidos}

Se explica las misiones y las tareas que hay en cada una de ellas, así como las acciones diarias a ejecutarse al inicio de cada clase. En este apartado, la plataforma tiende a sobresalir, debido a que al iniciar la clase de manera ineludible el profesor debe iniciar la clase con la ejecución de una dinámica, la misma selecciona a uno o varios alumnos e interactúa con ellos en temas relacionado con la clase. Por ejemplo, la plataforma preguntará al alumno sobre que se trató la clase pasada, a parte de las preguntas existen varias dinámicas listas para ser ejecutadas; por tal motivo, desde el comienzo se despertará y se mantendrá el interés de los estudiantes (Figura 2). Por tanto, el punto de vista didáctico tiene como objetivo apoyar en la disposición de los educadores para contribuir de manera positiva en el conjunto de operaciones en la respuesta que seda a los problemas en el aula, de igual manera los estudiantes aprenden las operaciones elementales de manera ludificada. 


\section{Figura 2.}

Ubicación de contenidos.

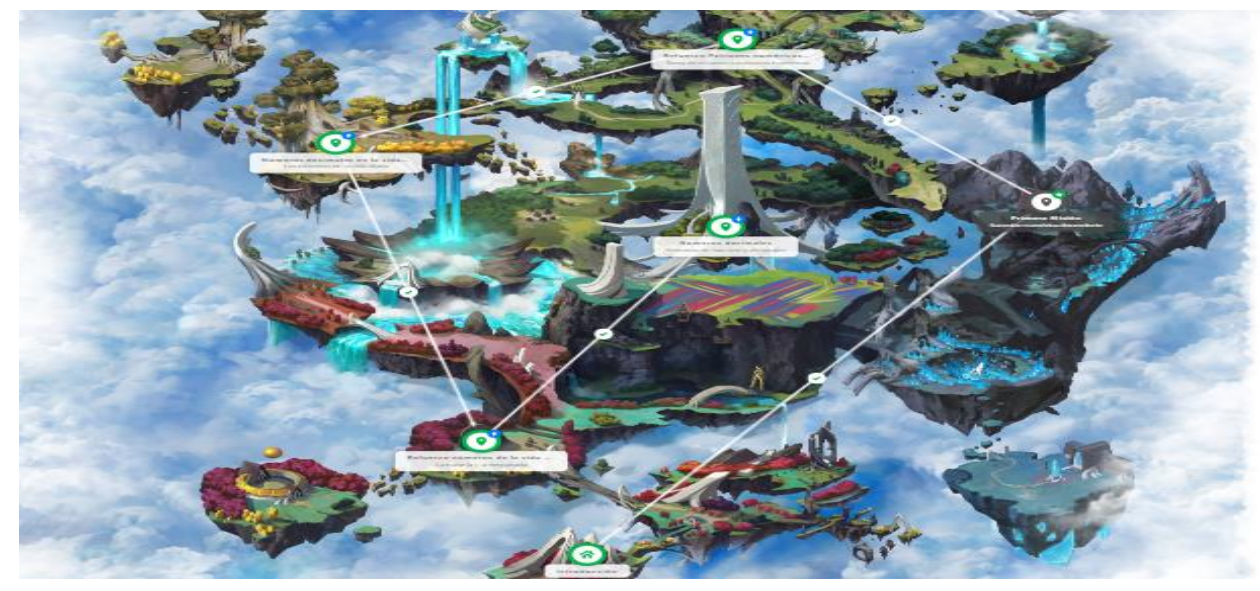

Fuente: https://game.classcraft.com/

Es aquí, donde cada estudiante percibe gran contenido de valor con respecto a su aprendizaje en las operaciones elementales, conforme su dedicación, su esfuerzo y perseverancia, la plataforma asigna bonos, monedas, o experiencia. Además, cuando sus participantes envían las tareas antes de la fecha asignada, los círculos verdes comprenden las misiones, al ingresar en la primera de ellas, se despliega un saludo de bienvenida, y explica de manera breve su funcionalidad. A su vez, las misiones se desbloquean de manera automática cada vez que el estudiante complete un reto, podrá visibilizar en su mapa el próximo desafío.

\section{Tercera fase. Desarrollo de contenidos}

En este apartado se explica sobre los puntos de experiencia que cada alumno obtiene si cumple con las tareas asignadas a tiempo, las actividades planificadas en la plataforma, así como exploraciones interactivas permiten a los alumnos vivir una experiencia diferente, gran parte de los contenidos han sido desarrollado en diversos aplicativos en 
línea, así como Genial.ly, y para reforzar lo comprendido se utilizó los formularios de Google y Educaplay (Figura 3).

\section{Figura 3.}

Desarrollo de contenidos.

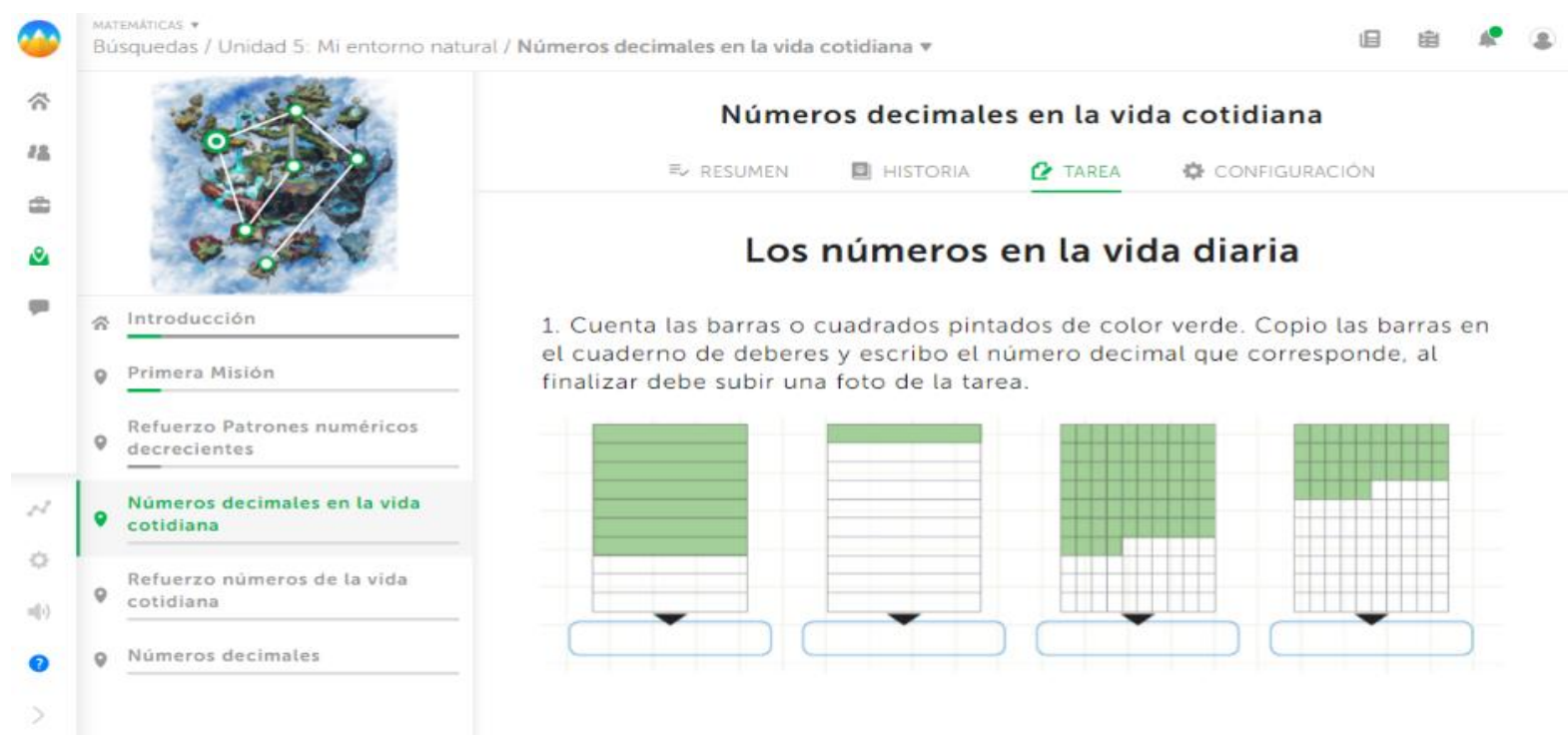

Fuente: https://game.classcraft.com/

Cada contenido está estructurado de manera que los estudiantes lo desarrollen y no sientan el sentimiento de la rutina. Por el contrario, cada reto para cada misión es diferente, es decir, a experiencia del alumno al sumergirse en la plataforma e interactuar en la misma da como resultado una mejora considerable y en la facilitación del proceso enseñanza-aprendizaje.

Cabe destacar el aspecto esencial en esta fase, es la incorporación de herramientas de clase como dinámicas previas al desarrollo de los contenidos del docente. Sin embargo, de una amplia gama de subniveles o actividades, la idónea para esta estrategia didáctica 
es la denominada "Los jinetes de Vay", con la cual se promueve una mayor participación de los estudiantes que posean bajos puntajes en la jornada educativa.

Similarmente, al finalizar la clase el docente debe implementar una actividad evaluativa de los conocimientos adquiridos durante esa jornada. Así, la actividad recomendable para esta estrategia es "La batalla de jefes", en la cual el docente asigna preguntas de forma individual o grupal, con la finalidad de obtener puntuaciones para cada uno de ellos y sumarlas a sus avatares o personajes.

Por otro lado, el docente tiene la opción de asignar puntuaciones en forma de recompensas a los estudiantes o equipos que contribuyan positivamente durante la jornada educativa, a través de la actividad denominada "La rueda del destino". A su vez, puede penalizarlos con una disminución de puntos si su comportamiento fue negativo. Lo que facilita y suscita la cooperación y el trabajo en equipo, así como el cambio de actitud en los estudiantes.

\section{Cuarta fase. Evaluación}

Para la última etapa dentro del Classcraft se elaboró una evaluación en Kahoot sobre la unidad 5, conformadas por interrogantes de verdadero y falso, seleccionando la respuesta correcta, entre otras. Como se ha visto en otras investigaciones al insertar herramientas tecnológicas para medir el aprendizaje sobre algún conocimiento, el estudiante responde mejor ante nuevos entornos, en dependencia sobre la efectividad de la gamificación, la evaluación revela datos con los cuales el docente podrá ajustar algún cambio o mejora en la plataforma de ser requerido. 


\section{Figura 4.}

Evaluación.

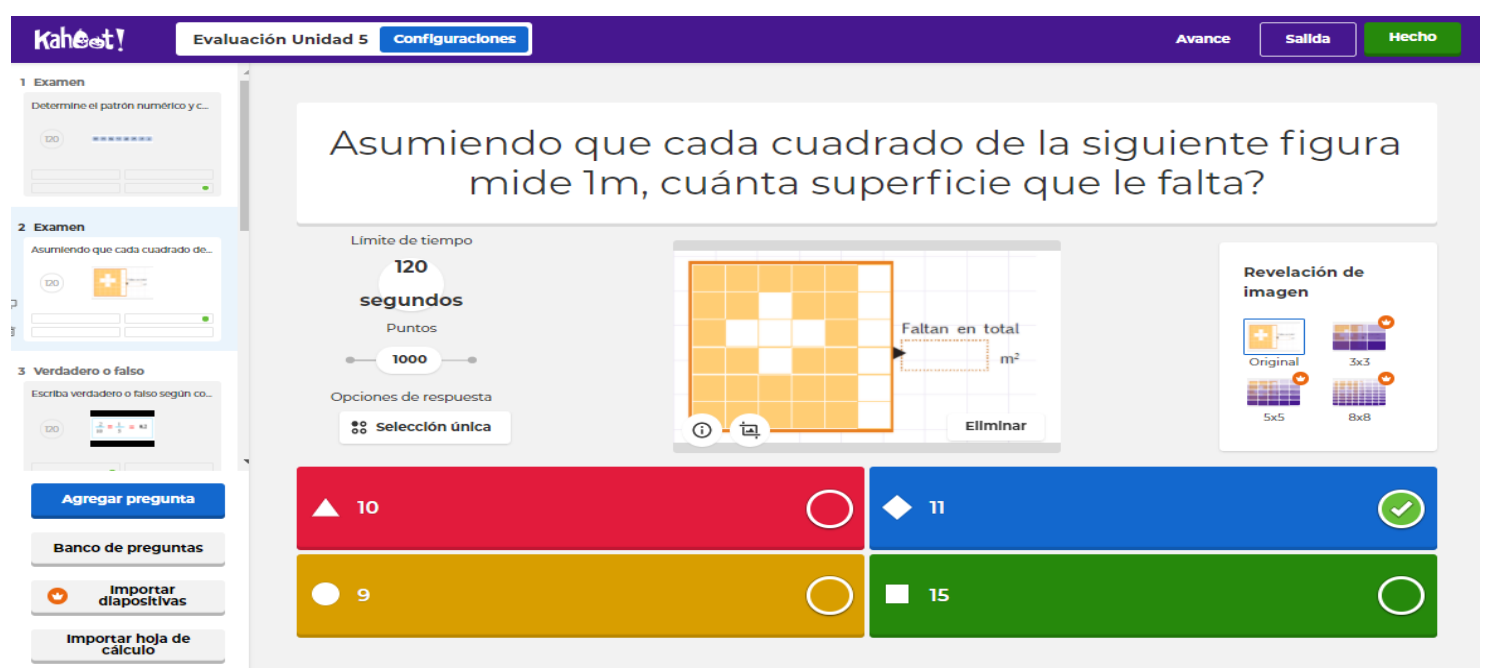

Fuente: https://game.classcraft.com/

Para la evaluación se tomó en consideración una herramienta digital freemium, llamada Kahoot, la cual lleva varios años en uso por muchos docentes para reforzar los contenidos impartidos, pero su principal utilización al momento de evaluar, permite a los estudiantes competir de manera sana por los primeros lugares. Cabe destacar, que se le puede utilizar para evaluar equipos de alumnos, esto desde luego genera una discusión y da paso al análisis, y a la emisión de criterios sobre sus posibles respuestas.

\section{Validación de la estrategia didáctica}

A continuación, se presenta la validación de la estrategia didáctica de gamificación a través de la técnica ladov, la cual evalúa la satisfacción de los educadores del área de educación básica respecto a la propuesta; seleccionándose a 10 de ellos, quienes mencionaron los siguientes resultados en las tres preguntas cerradas y en el índice de satisfacción individual (ISI): 
Tabla 2.

Resultados ISI ladov.

\begin{tabular}{|c|c|c|c|c|c|c|}
\hline \multirow[b]{2}{*}{ Docente } & \multicolumn{3}{|c|}{ Respuesta pregunta } & \multicolumn{3}{|c|}{ Resultado } \\
\hline & 1 & 2 & 3 & ISI & Escala & $\begin{array}{c}\text { Nivel de } \\
\text { satisfacción }\end{array}$ \\
\hline 1 & No & Sí & Me gusta mucho & 1 & $A$ & \multirow{6}{*}{$\begin{array}{c}\text { Clara } \\
\text { Satisfacción }\end{array}$} \\
\hline 2 & No se & Sí & Me gusta mucho & 2 & $\mathrm{~A}$ & \\
\hline 3 & No se & No se & Me gusta mucho & 2 & A & \\
\hline 4 & No & Sí & $\begin{array}{l}\text { Me gusta más de lo } \\
\text { que me disgusta }\end{array}$ & 2 & $A$ & \\
\hline 5 & No & Sí & Me gusta mucho & 1 & A & \\
\hline 6 & No & Sí & Me gusta mucho & 1 & A & \\
\hline 7 & No & No se & $\begin{array}{l}\text { Me disgusta más de } \\
\text { lo que me gusta }\end{array}$ & 3 & B & $\begin{array}{c}\text { Más satisfecho } \\
\text { que } \\
\text { insatisfecho }\end{array}$ \\
\hline 8 & No & Sí & Me gusta mucho & 1 & $A$ & $\begin{array}{c}\text { Clara } \\
\text { Satisfacción }\end{array}$ \\
\hline 9 & No & No se & $\begin{array}{l}\text { Me disgusta más de } \\
\text { lo que me gusta }\end{array}$ & 3 & B & $\begin{array}{c}\text { Más satisfecho } \\
\text { que } \\
\text { insatisfecho }\end{array}$ \\
\hline 10 & No & Sí & Me gusta mucho & 1 & $A$ & $\begin{array}{c}\text { Clara } \\
\text { Satisfacción }\end{array}$ \\
\hline
\end{tabular}

Fuente: Elaboración propia (2021).

En ese sentido, a nivel individual se denota una escala mayoritariamente de $A$, que equivale a una clara satisfacción de los estudiantes respecto a la estrategia didáctica. Aunque también, algunos estudiantes se sienten más satisfechos que insatisfechos predomina un nivel idóneo de complacencia. Posteriormente, se obtuvo un 0.90 como índice de satisfacción grupal (ISG) con su respectiva fórmula, asumiéndose que los docentes de Educación Básica tienen una clara satisfacción por la estrategia didáctica, en satisfacción al manejo de la gamificación en el proceso de enseñanza-aprendizaje y comprensión de operaciones elementales en el área de matemáticas. 


$$
\begin{gathered}
I S G=\frac{A(+1)+B(+0.5)+C(0)+D(-0.5)+E(-1)}{N} \\
I S G=\frac{8(+1)+2(+0.5)+0(0)+0(-0.5)+0(-1)}{10} \\
I S G=\frac{9}{10} \\
I S G=0.90
\end{gathered}
$$

Además, dentro de las dos preguntas abiertas los docentes señalan que la fase de descubrimiento de contenidos es aquella que más les agrada, pues el docente debe iniciar la clase con la ejecución de una dinámica, la misma que selecciona a uno o varios alumnos e interactúa con ellos en temas relacionado con la clase. De esta forma, el aprendizaje se realiza de forma didáctica, promoviéndose la resolución de problemas en entornos digitales y los estudiantes aprenden las operaciones elementales de manera ludificada. Sin embargo, dentro de los aspectos negativos de esta estrategia es su aplicabilidad, ya que algunos docentes que no tienen los conocimientos necesarios referentes al manejo de la herramienta tecnológica, por otro lado, existe en algunos miembros, una resistencia a migrar de una enseñanza tradicional a una digital. Por ello, es necesario que previo a la implementación de estas estrategias didácticas se realicen actividades de capacitación sobre todos en los aspectos de manejo de la herramienta y sus diferentes funcionalidades. 


\section{CONCLUSIÓN}

Al indagar sobre las nuevas formas de enseñanza-aprendizaje en la formación del área en concreto de matemáticas, se evidenciaron las bases teóricas sustentan la utilidad de la gamificación, las investigaciones realizadas apoyan la realización de una estrategia didáctica por medio de los recursos tecnológicos como una alternativa en la enseñanza de las operaciones elementales en estudiantes de quinto año de básica, así se consiguió evidenciar que diferentes estrategias lúdicas refuerzan el trabajo de los docentes con sus estudiantes.

El diagnóstico posibilitó evidenciar que las estrategias didácticas durante la enseñanza de las operaciones elementales que utilizan los docentes actualmente no han conseguido aportar resultados efectivos en sus estudiantes, a través de una encuesta con preguntas cerradas realizada a los alumnos, se registró en su totalidad el bajo interés en la asignatura. Por tal motivo, la metodología de la gamificación se origina con la finalidad de hallar opciones para modificar la situación actual y aportar como un instrumento de ayuda en los educandos cuyo propósito sea desarrollar el conocimiento sobre las operaciones elementales.

La estrategia didáctica está forjada en una plataforma virtual, dónde su propósito recae en transformar un proceso de enseñanza habitual e integrarlo como una oferta innovadora en la plantilla de docentes, por medio de actividades diarias y destrezas lúdicas. Como consecuencia fortifica los contenidos dotados por su docente que se evidencia en la satisfacción de los mismos con la estrategia propuesta; donde sus fases son cuatro, introducción de la estrategia, descubrimiento de contenidos, desarrollo de contenidos, y evaluación. Sin embargo, para su aplicación es pertinente una capacitación a los docentes, donde se familiaricen con el entorno de la herramienta y sus diferentes funciones. 


\section{FINANCIAMIENTO}

No monetario.

\section{AGRADECIMIENTOS}

A la Pontificia Universidad Católica del Ecuador, Ambato; por motivar el desarrollo de esta investigación.

\section{REFERENCIAS CONSULTADAS}

Aguilera, C., Santos, C., Pinargote, B. \& Erazo, J. (2020). Gamificación: estrategia didáctica motivadora en el proceso de enseñanza aprendizaje del primer grado de educación básica. [Gamification: motivating didactic strategy in the teachinglearning process of the first grade of basic education]. Revista Cognosis, 5(2), 5170. DOI: https://dx.doi.org/10.33936/cognosis.v5i3.2083.

Ardila, J. (2019). Supuestos teóricos para la gamificación de la educación superior. [Theoretical assumptions for gamification of higher education] Magis, Revista Internacional de Investigación en Educación, 12(24), 71-84. DOI: https://dx.doi.org/10.11144/Javeriana.m12-24.stge.

Classcraft. 2020. Classcraft. Recuperado 20 de octubre de 2020. Recuperado de: http://bit.ly/38z6ksN.

Constitución de la República del Ecuador. (2017). Plan Nacional de Desarrollo 20172021-Toda una Vida. [National Development Plan 2017-2021-A Lifetime]. Gobierno Electrónico de Ecuador. Recuperado de: https://www.gobiernoelectronico.gob.ec/normativa/.

Corchuelo-Rodríguez, C. (2018). Gamificación en educación superior: experiencia innovadora para motivar estudiantes y dinamizar contenidos en el aula. [Gamification in higher education: innovative experience to motivate students and stimulate content in the classroom]. Edutec. Revista Electrónica De Tecnología Educativa, (63), 29-41.DOI: https://doi.org/10.21556/edutec.2018.63.927 
Cruz-Pichardo, I. M., \& Cabero-Almenara, J. (2020). Una experiencia gamificada en el aprendizaje de los triángulos en geometría: grado de aceptación de la tecnología. [A gamified experience in learning triangles in geometry: degree of acceptance of technology]. Revista Prisma Social, (30), 65-87. Recuperado de: https://revistaprismasocial.es/article/view/3744

Díez, J., Bañeres, D. \& Serra, M. (2017). Experiencia de gamificación en Secundaria en el Aprendizaje de Sistemas Digitales. [Gamification experience in Secondary in Learning Digital Systems]. Education in the Knowledge Society (EKS), 18(2), 85105. DOI: https://dx.doi.org/10.14201/eks201718285105

Farias, D. \& Pérez, D. (2010). Motivación en la Enseñanza de las Matemáticas y la Administración. [Motivation in the Teaching of Mathematics and Administration]. Formación universitaria, 3, 33-40. DOI: https://dx.doi.org/10.4067/S0718$\underline{50062010000600005 .}$

García, F., Valls, C. \& Gisbert, M. (2018). Diseño e implementación de un cambio metodológico en el ámbito científico mediante la gamificación y el modelo de las 5E.[Design and implementation of a methodological change in the scientific field through gamification and the 5E model]. Edutec. Revista Electrónica de Tecnología Educativa, (66), 65-78. DOI: https://dx.doi.org/10.21556/edutec.2018.66.1187

García, M. y Neira, R. (2017). Análisis para la gamificación de un curso de Formación Profesional. [Analysis for the gamification of a Professional Training course]. IE Comunicaciones: Revista Iberoamericana de Informática Educativa, (26), 46-60. Recuperado de: https://scholar.google.com/citations?user=fSjuppYAAAAJ\&hl=en

Genial.ly (2020). Genially, the Tool for Bringing Your Content to Life. Genially. Recuperado de http://bit.ly/2LugiCW

Gilbert, R. \& Sarah, A. (1982). El ordenador en la escuela. [ The computer at school] Perspectiva: revista trimestral de educación, (12), 4 - Unesco Digital Library. DOI: https://bit.ly/35CwOYG.

González, E., García, D., Erazo, C. \& Erazo, J. (2020). Khan Academy: Estrategia metodológica para el aprendizaje de las gráficas de funciones trigonométricas. [ Methodological strategy for learning the graphs of trigonometric functions] $\begin{array}{llll}\text { EPISTEME KOINONIA, 3(6), } & \text { 78-101. }\end{array}$ https://dx.doi.org/10.35381/e.k.v3i6.816 
Hamari, J., Koivisto, J. \& Sarsa, H. (2014). Does Gamification Work? A Literature Review of Empirical Studies on Gamification. 2014 47th Hawaii International Conference on System Sciences, Waikoloa, HI, USA, 2014, 3025-3034. DOI: https://dx.doi.org/10.1109/HICSS.2014.377

Hammer, J. \& Lee, J. (2011). Gamification in Education: What, How, Why Bother? Academic Exchange Quarterly, 15, 1-5.

Holguín-García, F.; Holguín-Rangel, E.; \& García-Mera, N. (2020). Gamificación de la enseñanza de las matemáticas: una revisión sistemática. [Gamification of mathematics teaching: a systematic review]. Telos: revista de Estudios Interdisciplinarios en Ciencias Sociales, 22 (1), 62-75. DOI: www.doi.org/10.36390/telos221.05

Kuzmina, N. V. (1970). Metódicas investigativas de la actividad pedagógica. [Investigative methods of pedagogical activity ]. Moscú, Rusia: Editorial Leningrado.

Instituto Nacional de Estadísticas y Censos. (2017). Encuesta Multipropósito. [Multipurpose Survey]. Recuperado de www.inec.gob.ec

Macanchí-Pico, M., Bélgica Marlene O.., \& Campoverde-Encalada, M. (2020). Innovación educativa, pedagógica y didáctica. Concepciones para la práctica en la Educación Superior. [Educational, pedagogical and didactic innovation. Conceptions for practice in Higher Education]. Universidad y Sociedad, 12(1), 396-403.

Mallitasig, A. \& Freire, T. (2020). Gamificación como técnica didáctica en el aprendizaje de las Ciencias Naturales. [Gamification as a didactic technique in the learning of Natural Sciences]. INNOVA Research Journal, 5(3), 164-81. DOI: https://dx.doi.org/10.33890/innova.v5.n3.2020.1391.

Ministerio de Educación del Ecuador (2020). Pasa la voz. [Spread the word]. Recuperado de: https://educacion.gob.ec/pasa-la-voz/.

Mora, M. y Camacho, J. (2019). Classcraft: inglés y juego de roles en el aula de educación primaria. [Classcraft: English and Role Play in the Primary Education Classroom] Apertura (Guadalajara, Jal.).11(1), 56-73. DOI: https://dx.doi.org/10.32870/ap.v11n1.1433. 
Navarro, G. (2017). Tecnologías y nuevas tendencias en educación: aprender jugando. El caso de Kahoot. [Technologies and new trends in education: learning by playing. The case of Kahoot]. Opción: Revista de Ciencias Humanas y Sociales, (83), 25277.

Prieto M., Díaz, D., Sanz, M. \& Reyes, E. (2014). Experiencias de aplicación de estrategias de gamificación a entornos de aprendizaje universitario. [Experiences of applying gamification strategies to university learning environments] ReVisión, 7(2), 7. DOI: https://bit.ly/2L2Mf4R

Reglamento General a la Ley Orgánica de Educación Intercultural. (2017). Ley orgánica de Educación Intercultural. [Organic Law of Intercultural Education]. Recuperado de https://bit.ly/3oNRnYJ

Rodríguez-Cepeda, R. (2018) Los modelos de aprendizaje de Kolb, Honey y Mumford: implicaciones para la educación en ciencias. [Kolb, Honey, and Mumford's Models of Learning: Implications for Science Education]. Sophia 14(1); 51-64. DOI: http://dx.doi.org/10.18634/sophiaj.14v.1i.698

Sánchez, D., Langer, M. \& Kaur, R. (2020). Gamification in the Classroom: Examining the Impact of Gamified Quizzes on Student Learning. Computers \& Education, 144, 103666. doi: https://dx.doi.org/10.1016/..compedu.2019.103666

Torres, A., Romero, L., Amor, M. y Björk, S. (2018). Modelo Teórico Integrado de Gamificación en Ambientes E-Learning (E-MIGA). [Integrated Theoretical Model of Gamification in E-Learning Environments (E-MIGA)]. Revista Complutense de Educación, 29(1), 129-45. doi: https://dx.doi.org/10.5209/RCED.52117.

Unesco. (2014). Enseñanza y aprendizaje: Lograr la calidad para todos. [Teaching and Learning: Achieving Quality for All]. París, Francia: Organización de las Naciones Unidas para la Educación. Recuperado de: https://unesdoc.unesco.org/ark:/48223/pf0000225654 spa

Valda, F. \& Arteaga, C. (2015). Diseño e implementación de una estrategia de gamificacion en una plataforma virtual de educación. [Design and implementation of a gamification strategy in a virtual education platform]. Fides et Ratio - Revista de Difusión cultural y científica de la Universidad La Salle en Bolivia, 9(9), 65-80. doi: http://bit.ly/3nlY79Y. 
EPISTEME KOINONIA

Revista Electrónica de Ciencias de la Educación, Humanidades, Artes y Bellas Artes

Año IV. Vol IV. $N^{\circ} 7$. Enero - Junio. 2021

Hecho el depósito de Ley: FA2018000022

ISSN: 2665-0282

FUNDACIÓN KOINONIA (F.K).

Santa Ana de Coro, Venezuela

Danny Rafael Rosero-Guanotásig; Ricardo Patricio Medina-Chicaiza

C2021 por los autores. Este artículo es de acceso abierto y distribuido según los términos y condiciones de la licencia Creative Commons Atribución-NoComercial-Compartirlgual 4.0 Internacional (CC BY-NC-SA 4.0) (https://creativecommons.org/licenses/by-nc-sa/4.0/). 